Dating was emphasised by the presentation of three longer reviews, outlining recent progress. D. Walton (University of Oxford and McMaster University) described the measurements made of the strength of the Earth's magnetic field from small samples taken from dated Greek pottery, using a SQUID magnetometer. The resulting curve shows rapid fluctuations in the field strength which, apart from their general interest, he pointed out might eventually be useful for precise dating. This account was nicely contrasted by one from J. Pilcher (University of Belfast) who reviewed the latest story on radiocarbon calibration. Here the rapid fluctuations ('wiggles') in the calibration curve have been a source of contention for several years, but it seems that, with the development of more accurate measurements, an honourable compromise is being reached. The new possibilities opened to radiocarbon dating through the development of detection by accelerators were also reviewed. Several papers were devoted to the dating of calcitic cave deposits from the disequilibrium found in the daughter products of uranium incorporated into the calcite. This approach, which bridges the gap betwen postassium/argon and radiocarbon dating, is clearly becoming more useful. Technical papers on thermoluminescence dating of pottery, flint and calcite showed that the time when it can provide a problem-free dating service is still some way off. The same also applies to dating by amino acid racemisation.

Research into prospection methods has diminished somewhat over the years, most papers being application reports or describing advances in dataprocessing techniques. The analysis of artefacts remains a very strong centre of interest, however. The sourcing of such raw materials as jade, obsidian, sanukite, marble, as well as pottery and glass, from measurements of characteristic trace element concentrations (usually by neutron activation) and also of stable isotope ratios were described. The relationship of metal composition to the parent ore also was the subject of discussion, since the smelting process, and especially the addition of fluxes, can complicate this. Fortunately the excavation of mining sites, such as the Bronze Age site of Timna (in the Negev), in which ore, slag, smelted metal and artefacts are all available for study, can add much information to a perennially-disputed area. Although no radically new method or material for determining provenance has been introduced, the quality of work now able to exploit the general principles

R. E. M. Hedges is at the Research Laboratory for Archaeology, Oxford University.

\section{Unified theories?}

THE analogy to Maxwell's unification of electricity and magnetism and the so-called 'unified' theory of weak and electromagnetic interactions expressed in F. Close's report in Nature News and Views 277, 349; 1979) "an intellectual achievement comparable with Maxwell's unification of electricity and magnetism", is an overstatement. Because however this statement is amply and repeatedly expressed in all journals, and even in the general press, it should be emphasised that the latter theory is not a unified theory on the level of Maxwell's unification. There are two coupling constants $G$ and $e$ of the weak and electromagnetic interactions before the 'unification', and there are still two coupling constants (parameters) after the 'unification', only mixed. Moreover, a large number of new (unobserved) fields are introduced here, but not in Maxwell's case. Two

F. CLOSE REPLIES: In his book 'The First Three Minutes' Weinberg writes: "A field theory which unifies [my italics] (weak and electromagnetic) forces was proposed by myself .

and Salam". It is the continuing accumulation of data supporting this theory that leads me and others to be so optimistic about it. On the other hand I recognise that there are important predictions that are as yet untested, hence my reference to it still as a candidate theory.

If the theory is eventually confirmed to be truly unified (in the sense of involving only one coupling constant,) then I would regard that as an achievement comparable to Maxwell's. That is a personal opinion which Professor Barut might not share. However he is correct to remind us that the unification (as defined above) is not yet confirmed

already established has considerably improved.

The original technical divisions of archaeology into Stone, Bronze and Iron Ages have become greatly refined as a result of more subtle studies of the materials manufactured in antiquity. Most studies centre on the interpretation of chemical analytical data, generally obtained by non (or minimally) destructive methods. These are enhanced by such sophisticated techniques as ESR, and Mössbauer spectrometry, from which, for example, information about the firing conditions of pottery, and possibly subsequent weathering on burial, is obtained. Several papers were given on coin analyses, as well as considerations on distributions of coin dies. The study of ancient metallurgy bene- general observations may be made.

(1) Given any two theories, one can always consider them together as part of a larger more symmetric system, if enough new fields (such as gluons, Higgsons) are introduced. It is like putting any two broken shapes into a larger more complex or symmetrical figure if enough clay and glue is introduced. (2) A theory is the more renormalisable the more fields are present to cancel the divergences.

Under these circumstances gauge theories can be called unified field theories only if and when all these additional hypothetical gluons and Higgsons are found and if there is only one coupling constant. Until then all so-called 'evidence' must remain circumstantial and we should call them simply gauge theories and not unified theories.

A. O. BARLT

International Centre for Theoretical Physics, Trieste

even though no evidence against it has yet been found. The unified theory predicts that charged and neutral weak radiation exist and the corresponding quanta are $\mathrm{W} \pm$ and $\mathrm{Z}$ bosons with well defined masses. Just as the prediction of electromagnetic radiation was pivotal in electromagnetic theory so are the predictions of the masses of the weak quanta significant tests for the unified theory.

It is true that the properties of the Higgs fields are as yet unclear but I think that Professor Barut exaggerates by claiming that a "large number" of new unobserved fields are introduced. The inclusion of gluons in his list is particularly misleading. These have nothing to do with the weak-electromagnetic unification but are instead the gauge fields of QCD-the candidate theory of the strong force.

fits greatly from the information available from metallography. Thus we were able to consider how much the workers of Early Dynastic Egypt knew of the work-hardening properties of their copper tools, and how much Mediaeval armourers understood of the tempering properties of their steels. Modern experiments with reconstructed bows have confirmed theories of armourpiercing, and show that Mediaeval craftsmen, while perhaps not aware of the theory, nevertheless achieved optimum design in practice.

The overall impression left was of an encouraging increase in the quality of work presented. On the whole the archaeology considered was more appriate and interpretations better judged. 